with radius increments of $11 \mathrm{~mm}$ gave a real value of $0.2 \mathrm{~mm}$. Because of the small size of the photographs only a few circles could be drawn, but the results (Fig. 8) show that a tighter packing is produced in the claybearing sandstone. The photographs are too small to give very good results, but with sufficient centres chosen from a large picture and a careful choice of radius increment for the reference circles, packing textures could be easily and conveniently determined.

I thank Ted Willett and Valerie Smalley, both of this College, for their assistance.
${ }^{1}$ Pettijohn, F. J., Sedimentary Rocks, second ed., 13 (Harper, New York, 1957).

2 Krumbein, W. C., and Pettijohn, F. J., Manual of Sedimentary Petrography (Appleton, New York, 1938).

${ }^{3}$ Graton, L. C., and Fraser, H. J., J. Geol., 43, 785 (1935).

4 Smalley, I. J., Math. Mag., 36, 295 (1963).

5 Smalley, I. J., Amer. J. Sci., 262, 242 (1964).

'Morell, W. E., and Hildebrand, J. H., J. Chem. Phys., 4, 224 (1936).

${ }^{7}$ Scott, G. D., Nature, 194, 956 (1962).

8 Prins, J. A., Naturwiss., 19, 435 (1931).

${ }^{9}$ Steers, J. A., The Coastline of England and Wales, 320 (Camb. Univ. Press,

${ }^{10}$ Heald, M. T., J. Geol., 64, 16 (1956).

\title{
SEMICONDUCTION IN KAOLIN
}

\author{
BY A. K. CHATTERJI \\ Central Building Research Institute, Roorkee, India
}

$I^{\mathrm{T}}$ has been reported that the electrical conductivity of kaolin increases with temperature ${ }^{1}$. This observation indicates that kaolin may be a semiconductor. In absence of usual confirmatory experiments, an attempt is made here to ascertain the semiconducting nature of kaolin from less direct evidence as adsorption of lime by kaolin in presence of water and rheology of dilute aqueous suspensions of kaolin. The possibility that kaolin may be a ' $p$ ' type semiconductor is indicated.

From the results of measurements on the adsorption of lime at ordinary temperatures by kaolin, kaolin heated at $800^{\circ} \mathrm{C}$ with or without addition of zinc oxide and of electrical conductivity of these materials at different temperatures, given elsewhere ${ }^{1,2}$, it will be seen that at any temperature the electrical conductivity of kaolin, previously heated with zinc oxide, is lowest and that of unfired kaolin is highest. ${ }^{1}$. Amount of lime chemisorbed from saturated lime water at ordinary temperatures, in a given time, is highest in kaolin fired with zinc oxide and least in unfired kaolin ${ }^{2}$. This antiparallel behaviour of lime adsorption and electrical conductivity means that the reaction between lime and kaolin in the presence of water is a semiconductor surface reaction which may either be an ' $n$ ' typo reaction on ' $p$ ' typc semiconductor or vice versa depending on the type of semiconduction of kaolin.

It is stated that, at the temperatures worked with, kaolin previously fired at $800^{\circ} \mathrm{C}$ has higher conductivity than kaolin which has been similarly fired with zinc oxide ${ }^{1}$. Thus on addition of an ' $n$ ' type semiconducting oxide $(\mathrm{ZnO})$ the conductivity of fired kaolin is lowered. This may occur, if the semiconduction in fired kaolin is predominantly by 'holes', through the combination of these with the electrons introduced by $\mathrm{ZnO}$. Again, kaolin fired at $800^{\circ} \mathrm{C}$ has lower conductivity than unfired kaolin'. This may indicate that the effect of dehydration is to remove 'holes' from unfired kaolin (dehydration taking place through transfer of 'holes' from the erystal). Thus, kaolin may be a ' $p$ ' semiconductor itsolf; effect of dehydration and heating it with ' $n$ ' type semiconducting oxide is to change it into a more and more ' $n$ ' type semiconducting oxide.

In a recent communication ${ }^{3}$ the rheological behaviour of dilute aqueous suspensions of some reactive solids, as lime, portland cement and plaster of paris, has been explained on the assumption that the reaction between these materials and water is a chemisorption process. In such a process the position of the bulk Fermi-level in the particles controls the coverage of chemisorbed molecules. The coverage determines the volume over which stabilization of water lattice takes place and the viscosity of the suspension depends on the extent of the volume over which water structure is stabilized. It is concluded that the rheological behaviour of such suspensions is controlled by the position of bulk Fermi-level in the particles in suspensions. Again, plasticity of clay-water systems has been explained on the assumption that in such a system a layer of water is rigidly attached on the surface of the clay mineral ${ }^{4}$. The nature of the interaction between the water layer and the clay mineral surface is, however, uncertain. Assuming that a chemisorption process is occurring, hydroxyl groups may get attached on the surface of the clay mineral through a transfer of electrons from the crystal (' $n$ ' type reaction) as in the reactions between portland cement, clinker and water ${ }^{6}$, and lime and water? ${ }^{7}$. If it were so, then the rheological behaviour of dilute suspensions of kaolin in water would be controlled by the position of bulk Fermi-level in the crystal: viscosity will increase or decrease as the position of the Fermi-level is raised or lowered. For the present purpose ono has to know what would happen to the viscosity of aqueous suspensions of kaolin which has been previously allowed to react with lime in the presence of water. It has been found that dilute aqueous suspensions of kaolin, so treated and then washed and dried, have lower plastic viscosity than similar suspensions of $\mathrm{H}$-kaolin ${ }^{5}$. This means that, on reacting with lime, Fermi-level of kaolin has been lowered. This can only happen when the reaction between lime and kaolin in the presence of water is an ' $n$ type reaction. With the reaction type settled, the antiparallel behaviour of lime adsorption and electrical conductivity would mean that kaolin is a ' $p$ ' type semiconductor, provided the assumption regarding the nature of interaction between kaolin and water is correct.

The electrical nature of kaolin appears to be similar to that of portland cement clinker. Possibility of the latter behaving as a ' $p$ ' type semiconductor has been indicated elsewhere ${ }^{6}$. The hydraulic action of cement clinker also increases with the decrease of electrical conductivity of clinker ${ }^{6}$ as in the case of adsorption of lime by kaolin in the presence of water. Parallelism in the nature of these reactions is clear. The Fermi-level appears to be the controlling factor.

It is concluded that there is a possibility that kaolin may be a ' $p$ ' type semiconductor and that some of its physical and chemical properties may be controlled by the position of bulk Fermi-level in it. The latter suggestion should also be true for other silicates which may prove to be semiconductors. This approach, then, will open a new aspect of chemistry and technology of such silicates.

${ }^{1}$ Chatterji, A. K., Nature, 192, 1180 (1961).

2 Chatterji, A. K., Phatak, T. C., and Dhariyal, K. D., J. Sci. Indust. Res. (India), 19B, $493(1960)$.

${ }^{3}$ Chatterji, A. K., and Kapse, G. W., Nature, 200, 868 (1963).

' Chatterji, A. K., Nature, 181, 1118 (1958).

${ }^{5}$ Chatterji, A. K., and Kapse, G. W. (unpublished results).

Chatterji, A. K., and Phatak, T. C., Nature, 203, 138 (1964).

${ }^{7}$ Chatterji, A. K., Phatak, T. C., and Dhariyal, K. D., Nature, 203, 61 (1964). 